MG4

2:30pm $-2: 45 p m$

\title{
SILICON RESONANT CAVITY ENHANCED 12X1 PHOTODETECTOR ARRAYS FOR OPTICAL INTERCONNECT RECEIVER INTEGRATION
}

\author{
Matthew K. Emsley, Olufemi I. Dosunmu, 'Paul Muller, ${ }^{1}$ Yusuf Leblebici, M. Selim Uniu
}

Boston University College of Engineering, 8 Saint Mary's St. Boston MA 02215 USA, memsley@bu.edu

${ }^{1}$ Swiss Federal Institute of Technology, Lausanne Switzerland

High bandwidth short distance communications standards are being developed based on parallel optical interconnect fiber arrays to meet the needs of increasing data rates of inter-chip communication in modern computer architecture. To ensure that this standard becomes an attractive option for computer systems, low cost components must be implemented on both the transmitting and receiving end of the fibers. To meet this low cost requirement all-silicon based receiver circuits are actively being pursued [1], however, manufacturing high speed, high efficiency silicon photodetectors presents a technical challenge.

Resonant cavity enhanced (RCE) photodetectors have been shown to provide the required bandwidth-efficiency product but have remained a challenge to reproduce through commercially available fabrication techniques. We have previously developed a method to produce silicon wafers with high reflectance buried distributed Bragg reflectors (DBR) [2]. The substrates consist of a twoperiod, $90 \%$ reflecting, DBR fabricated using a double silicon-on-insulator (SOI) process. Discrete RCE Si photodetectors have previously been fabricated with $40 \%$ quantum efficiency at $860 \mathrm{~nm}$, a FWHM of $25 \mathrm{ps}$, and a $3 \mathrm{~dB}$ bandwidth in excess of $10 \mathrm{GHz}$ [3].

In this paper we present Si RCE $12 \times 1$ photodetector arrays that have been fabricated and packaged with silicon based amplifiers to demonstrate the feasibility of a low cost monolithic silicon photoreceiver array. The 12xl photodetector arrays were designed for the PAROLI product line specification by Infineon [4]. The specification calls for 12 photodetectors with between 30 and $80 \mu \mathrm{m}$ photodetector active area in a linear array on a $250 \mu \mathrm{m}$ pitch. The fabricated $12 \times 1$ photodetector array can be seen in Fig. 1.

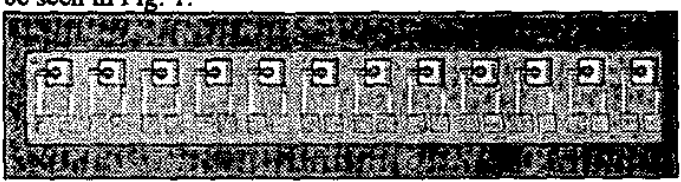

Fig. $112 \times 1$ photodetector array

The photodetectors consist of vertical Si RCE p-i-n photodiodes, a cross section is shown in Fig. 2, fabricated through standard Si processing techniques on double SOI reflecting wafers. The photodetectors have a $42 \mu \mathrm{m}$ detection area. Several samples were prepared for packaging with Helix HXR2312 $3.6 \mathrm{GHz} \quad 12$ Channel Receiver arrays. The samples were sent to Helix in Zurich, Switzerland and were bonded to the Helix receiver array using $25 \mu \mathrm{m}$ Gold wire wedge bonding. There were limitations in using this device but it was the only option for this testing. The first and perhaps most important limitation is the mismatch input impedance of the Helix amplifier. The Helix amplifier is designed to have a matched capacitive load of $450 \mathrm{fF}$, the typical capacitance of Optospeed InGaAs photodetectors which is the standard photodetector used in the optical interconnect standard. Our photodetectors have a $70 \mathrm{fF}$ capacitance. One other draw back is that even though the specification mentioned earlier called for 30 to $80 \mu \mathrm{m}$ diameter photodetectors the testing was done with $50 \mu \mathrm{m}$ core multi-mode fiber (MMF). This means that the $42 \mu \mathrm{m}$ photodetector was only capturing $70 \%$ of the incident light at best.

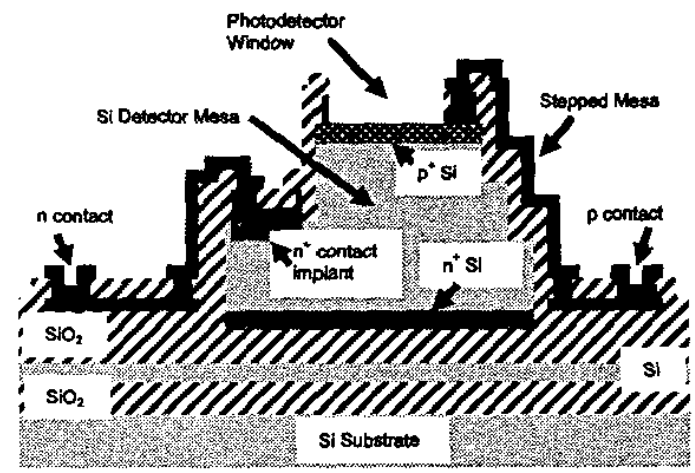

Fig. 2 Cross section schemtaic of Si RCE $p-i-n$ photodiode

The photodetectors were tested with the Helix receiver to measure the bit error rate BER and the eye-diagram. The photoreceiver was tested using an HP8664A Synthesized Signal Generator ${ }^{-}$connected to a HP70841B Pattern Generator which was connected to a laser driver and a New Focus Model-1780 850 $\mathrm{nm}$ 10G Hz Laser Diode, which was coupled to MMF and sent through an EXFO FVA-3100C Variable Attenuator. The output fiber of the Variable Attenuator was butt-coupled with the photodetector by line of sight and by maximizing the received photocurrent. The receiver is then connected to a HP70842B Error Detector to measure BER and a Agilent infinium DCA Wide Bandwidth Oscilloscope with HP54743A TDR Module to measure the eye-diagram. During the test the photodetector is biased at $1.8 \mathrm{~V}$ reverse bias.

Typically eye diagrams are measured on a photodetector connected to a transimpedance amplifier with a fixed gain. Therefore at higher frequencies a lowering of the output magnitude will occur due to $3 \mathrm{~dB}$ roll off. The Helix HXR2312 has a rail-to-rail limiting 
amplifier built in which means the magnitude of the output is constant for changing input magnitudes. Therefore the usefulness of the eye diagrams is questionable in this study as it can only reasonably measure the lateral eye opening due to diffusing carriers. Figure 3 shows the eye-diagram for the same randomly selected detector in a $12 x 1$ array used in the BER results. The eye shows good opening at $3 \mathrm{GHz}$ and $-9.5 \mathrm{dBm}$ optical power. The plot does not show any characteristic signs of long diffusion tails.

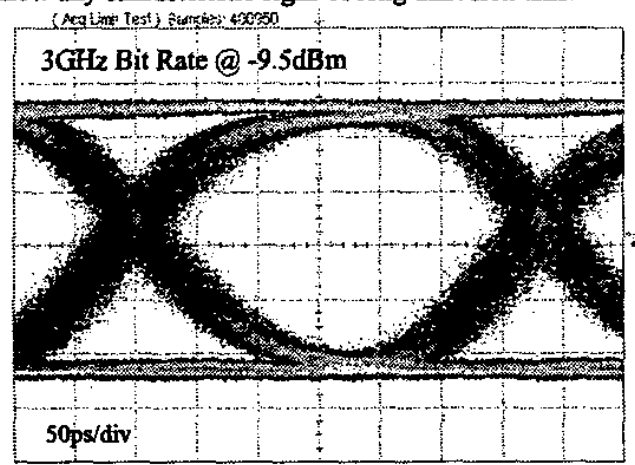

Fig. 3 Eye diagram for single device in $12 \times 1$ photodetector array at 3GHz

The HP70841B Pattern Generator was setup to send a PRBS 223-1 sequence to laser diode with a clock frequency of $1 \mathrm{GHz}, 1.5 \mathrm{GHz}, 2 \mathrm{GHz}$ and $3 \mathrm{GHz}$ on four separate runs. BER was measured using the HP70842B Error Detector which had the same pseudo random bit sequence (PRBS) 223-1 programmed for comparison. The BER was measured as a function of power by controlling the attenuation factor on the calibrated EXFO FVA-3100C Variable Attenuator. Figure 4 shows the measured BER from a randomly selected detector in a $12 \times 1$ array. Ideal "error free" transmission is considered to be 1 part in 1 trillion or a BER of $10-12$. At $3 \mathrm{GHz}$ the $\mathrm{Si}$ RCE photodetector was able to achieve a BER of 10.12 at $6.5 \mathrm{dBm}$ an order of magnitude worse than Optospeed's InGaAs photodetectors which require $-16.5 \mathrm{dBm}$ at the same BER. The other thing to note in Fig. 4 is the $5.5 \mathrm{dBm}$ shift in minimum received power for $1 \mathrm{GHz}$ transmission to $3 \mathrm{GHz}$ transmission. The designers at Helix felt that this shift in minimum received power is due to the mismatched load of the receiver on the photodetector caused by the lower capacitance. The other cause of an increase in minimum received power, at all frequencies, is the problem of not collecting all the light from the MMF as well as the reduced responsivity at $850 \mathrm{~nm}$ because of misaligned spectral peak.

This work has presented Si RCE $p$-i-n photodetector arrays designed for use in parallel optical interconnect systems. Photodetector arrays were wire bonded with existing $\mathrm{Si}$ based receiver circuits to make an all-Si photoreceiver operating at $3 \mathrm{GHz}$, showing performance that could soon compete with existing compound semiconductor photodetectors for a fraction of the cost. The wafers could also be used to fabricate a host of $\mathrm{Si}$ based integrated optoelectronics owing to the availability of Si processing and the availability of large wafer sizes. These wafers are well suited for large scale integration and are compatible with standard CMOS processing making them ideal for fabricating photodetectors monolithically with receiver circuits.

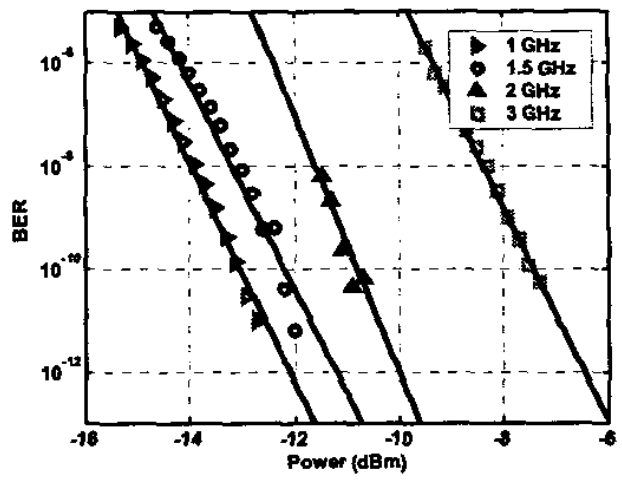

Fig. 4 BER measurment for single device in 12r1 photodetector array

\section{Acknowledgements}

The authors wish to thank Mike Ameen and Mark Harris at Axcelis Technologies for providing ionimplantation services for this project. We would also like to thank Philippe Flückiger and the entire staff at the Center of Microtechnology at EPFL for help in the fabrication of these detectors. Additionally we would like to thank Bruno Ghyselen of SOITEC SA for fabrication of the double SOI wafers. The authors also thank Martin Bossard and Jorg Wieland of Helix AG for packaging our detectors with their receiver circuits as well as for doing the BER and Eye-diagram testing.

This research was sponsored by the Army Research Laboratory (ARL) and was accomplished under the ARL Cooperative Agreement Number DAAD17-99-2-0070. The views and conclusions contained in this document are those of the authors and should not be interpreted as representing the Laboratory or the U.S. Government.

\section{References}

[1] B. Yang, J. D. Schaub, S. M. Csutak, D. L. Rogers, and J. C. Campbell, "10-Gb/s All-Silicon Optical Receiver," IEEE Photonics Technology Letters, Vol. 15, No. 5, May 2003, pp. 745-747.

[2] M. K. Emsley, O.I. Dosunmu, and M. S. Ửnlu, "Silicon Substrates With Buried Distributed Bragg Reflectors for Resonant Cavity-Enhanced Optoelectronics," IEEE Journal of Selected Topics in Quantum Electronics, Vol. 8, No. 4, July/August 2002, pp. 948-955.

[3] M. K. Emsley, O.I. Dosunmu, and M. S. Ũnla, "HighSpeed Resonant-Cavity-Enhanced Silicon Photodetectors on Reflecting Silicon-on-Insulator Substrates," IEEE Photonics Technology Letters, Vol. 14, No. 4, April 2002, pp. 519-521.

[4] Infineon Tecnologies, "Parallel Optical Links (PAROLD," www.infineon.com/paroli/, July 2003. 